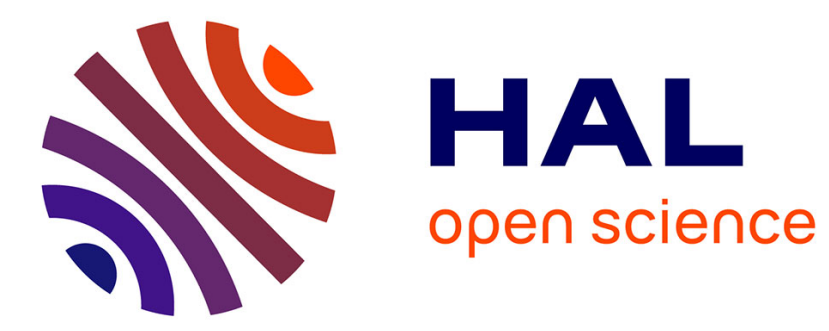

\title{
Voyage dans la galaxie décroissante
}

Fabrice Flipo

\section{To cite this version:}

Fabrice Flipo. Voyage dans la galaxie décroissante. Mouvements : des idées et des luttes, 2007, 50, pp.143-151. 10.3917/mouv.050.0143 . hal-00957890

\section{HAL Id: hal-00957890 https://hal.science/hal-00957890}

Submitted on 13 Mar 2014

HAL is a multi-disciplinary open access archive for the deposit and dissemination of scientific research documents, whether they are published or not. The documents may come from teaching and research institutions in France or abroad, or from public or private research centers.
L'archive ouverte pluridisciplinaire HAL, est destinée au dépôt et à la diffusion de documents scientifiques de niveau recherche, publiés ou non, émanant des établissements d'enseignement et de recherche français ou étrangers, des laboratoires publics ou privés. 


\section{Voyage dans la galaxie décroissante}

La décroissance : le terme interpelle, pose question, se retrouve dans des endroits improbables et prend bien des gens au dépourvu. Nous en traçons ici un petit panorama descriptif, en attendant d'entrer dans le détail des problématiques qui sont ainsi mises à l'agenda.

Fabrice Flipo

La décroissance gagne en audience à gauche. Ce terme, utilisé par J. Grinevald pour traduire le titre de l'ouvrage de Nicholas Georgescu-Roegen ${ }^{1}$, dont le titre original était The Entropy Law and the Economic Process ${ }^{2}$, n'était connu que de groupes d'experts quand le journal « La décroissance », né en mars 2004, a relancé ce concept dans l'espace public. Le journal tire à 45000 exemplaires, dont 25000 vendus. Il a doublé sa fréquence de parution, en devenant mensuel. Plusieurs sites ouebes sont consacrés à la décroissance. Decroissance.info est un site autogéré par des groupes locaux. Decroissance.net est le site officiel de l'Institut d'Etudes Economiques et Sociales pour la Décroissance Soutenable, de même tendance que le journal La Décroissance. Ladecroissance.org est le site du journal La Décroissance. Des «marches pour la décroissance » ont eu lieu à divers endroits (Lyon à Magny-Cours en juin 2005, en Loire-Atlantique, dans le Nord-Pas-de-Calais et en Charente-maritime) et d'autres sont en projet.

La décroissance a même son parti : le Parti pour la Décroissance (PPLD), né le 8 avril est né à Dijon. Il appelle à «une décroissance fondée sur l'équité, la soutenabilité et les valeurs humanistes, démocratiques, républicaines, non-violentes, défendants les droits de l'Homme et combattant toutes les formes de discrimination et de totalitarisme » De nombreux petits journaux soutiennent cette idée de décroissance : l'âge de faire, Silence etc. La décroissance circule beaucoup sous le manteau, elle génère des débats qui favorisent une réappropriation symbolique de notre milieu. Nicolas Ridoux, ingénieur, a synthétisé l'ensemble des éléments de la décroissance dans un petit livre très bien fait ${ }^{3}$.

1 Demain la décroissance: Entropie-écologie-économie, préface et traduction d'I. Rens et J. Grinevald, Lausanne, Pierre-Marcel Favre, 1979, 2e éd. revue et augmentée, La décroissance, Paris, Sang de la terre, 2006.

2 Harvard University Press, 1971.

3 N. Ridoux, La Décroissance pour tous, éditions Parangon, 2006. 
La décroissance n'est pas limitée à la presse à petit (ou moyen) tirage. L'édition du 25 mars du Monde 2, supplément du quotidien Le Monde, a consacré sa une et son dossier principal à la décroissance ${ }^{4}$. Ce thème a eu droit à plusieurs articles dans Le Monde ou ailleurs. On peut trouver des dossiers sur le sujet dans des lieux aussi improbables que TGV Magazine. La vague est profonde et tout laisse penser qu'elle sera durable.

Elle a commencé à toucher les grands partis politiques. Yves Cochet a poussé cette idée au sein des Verts mais il n'a pas remporté l'investiture pour la présidentielle. La motion «Utopia », emmenée par Frank Pupunat, est la première à avoir remis en cause la religion de la croissance au PS. Elle a obtenu 1,05\% des suffrages au congrès du Mans en novembre 2005. Le réseau des objecteurs de croissance, qui ne sont pas forcément des partisans de la décroissance, a été très actif autour de la candidature unitaire à gauche. Nicolas Hulot, conseillé entre autres par Jean-Paul Besset, a été présenté par Politis comme le croisé de la décroissance ${ }^{5}$ - et dénoncé par La Décroissance comme un " écotartuffe ». Corinne Lepage a réfuté cette idée, qu'elle juge trop négative, et Dominique Voynet a préféré la « décroissance de l'empreinte écologique », ce qui est très différent.

La revue Entropia a été lancée en novembre 2006 en présence d'une bonne cinquantaine de personnes à l'Assemblée Nationale pour aider à donner une consistance théorique à l'idée de décroissance. Le comité de rédaction est composé de Jean-Paul Besset ${ }^{6}$, J.-C. Besson-Girard ${ }^{7}$, François Brune ${ }^{8}$, Alain Gras ${ }^{9}$, Serge Latouche ${ }^{10}$ et Agnès Sinai ${ }^{11}$. La revue se présente comme « revue d'étude théorique et politique de la décroissance »: «Entropia s'inscrit dans la longue tradition de la revue d'idées et d'engagement, lieu d'expression privilégié d'une pensée collective naissante et qui s'élabore au fil du temps. Une pensée sur la crête des interrogations fondamentales de notre époque, pour l'amplification de la prise de conscience

4 Peut-on continuer comme ça ? Pollution-inégalités-énergie, la théorie de la décroissance revient, 25 mars 2006.

$5 \quad$ Edition du 9 novembre 2006

6 Ancien rédacteur en chef du Monde, coordinateur du «Pacte écologique » de Nicolas Hulot, auteur de « Comment ne plus être progressiste... sans devenir réactionnaire » chez Fayard

7 Paysan, artiste, auteur de « Decrescendo Cantabile » chez Parangon

8 Auteur de « Les médias pensent comme moi », " De l'idéologie aujourd'hui » etc.

9 Professeur d'anthropologie à Paris 1-Sorbonne, auteur de « La fragilité de la puissance » chez Fayard

10 Economiste, auteur du «Pari de la décroissance » (Fayard, 2006), « Survivre au développement » et bien d'autres ouvrages

11 Journaliste, co-auteure de « Sauver la Terre » avec Yves Cochet 
d'une situation de la condition humaine sans précédent, pour l'enrichissement de l'imaginaire théorique, poétique et politique de l'après-développement $»^{12}$. Le titre de la revue, «Entropia », ne fait pas référence à l'entropie au sens physique, ce concept remis au goût du jour en économie par Nicholas Georgescu-Roegen mais au grec « entropè », action de se retourner, regarder par-devers soi afin de contempler le chemin parcouru et prendre le temps de se demander s'il ne vaudrait mieux pas en changer.

Pour Entropia, «toute pensée qui refuse son autocritique n'est plus une pensée, mais une croyance. Elle quitte le terrain solaire de la lucidité pour les mirages de l'espérance. Depuis plus de cinquante ans, "la croissance» et «le développement» relèvent de ce statut irrationnel et dogmatique. Dans les années soixante-dix, cependant, quelques chercheurs hétérodoxes et que la clairvoyance n'effrayait pas (Illich, Georgescu-Roegen, Ellul, Partant, Castoriadis...) se sont dressés contre cette dictature de l'économisme et ont jeté les bases d'une pensée de la décroissance. Pensée dérangeante s'il en est. Depuis quelques années seulement, et singulièrement depuis le colloque intitulé : "Défaire le développent, refaire le monde » (UNESCO 2002), des publications comme Silence et l'Écologiste, le bulletin de « La Ligne d'horizon, les amis de François Partant ", lui ont fait une place grandissante dans leurs colonnes. Le bimestriel La Décroissance contribue, depuis trois ans, à accentuer son caractère iconoclaste et provocant. Car cette notion de décroissance bouleverse en effet les signes et les lignes : les signes théoriques et symboliques de reconnaissance comme les lignes des clivages politiques traditionnels. Cette situation peut engendrer des dérapages et des dérives théoriques et politiques qui exigent la plus grande vigilance de la pensée et des pratiques. $\rangle^{13}$

Et de fait des dérapages se sont produits. Des textes issus des théoriciens de la décroissance ont été retrouvés sur le site du Grece, dont les accointances avec l'extrême-droite sont de notoriété publique. Cela a jeté la confusion au sein des promoteurs de la décroissance, un peu moins au sein des théoriciens. Le Grece est en effet coutumier de la récupération d'idées sans l'aval de ses auteurs, le MAUSS en avait déjà fait l'expérience autrefois.

Au-delà des fausses polémiques, le travail ne manque pas. Les chantiers ouverts par la décroissance sont aussi nombreux que les manières de se servir de ce terme ou de s'y référer.

12 Entropia $\mathrm{n}^{\circ} 1$, novembre 2006.

13 Entropia ${ }^{\circ} 1$, novembre 2006. 
«Mot-obus » pour les uns, comme Paul Ariès ${ }^{14}$, servant à briser les lignes conceptuelles adverses, qui n'imaginent aucun avenir hors de la croissance, la décroissance peut aussi être élevée au rang de concept, en particulier dans le domaine économique. La décroissance désigne aussi une manière d'être, que l'on rencontre parfois sous le vocable de «simplicité volontaire $»$.

Les échanges entre Hervé Kempf ${ }^{15}$, Nathalie Kosciusko-Morizet ${ }^{16}$ et Sylvia Pérez-Vitoria ${ }^{17}$ dans l'émission « Du grain à moudre » sur France Culture le 1er février dernier montraient bien l'étendue du débat qui a été ouvert par la décroissance. Hervé Kempf a remis la question du luxe sur la place publique. S'appuyant sur l'économiste Thorstein Veblen, il a émis l'hypothèse que les comportements sont mus par l'ostentation plutôt que par les besoins, aussi la diffusion du modèle occidental dans le monde est-il la clé de la crise écologique mondiale. Nous, Occidentaux, devrions donc au plus vite pratiquer la sobriété, à commencer par les plus riches. La députée de l'Essonne, tout en reconnaissant l'urgence, a minimisé la responsabilité des riches. Soutenue par le journaliste Brice Couturier, qui en a perdu son devoir de neutralité, elle a cherché à démontrer que la croissance profitait aux plus pauvres, car la phase de croissance des pollutions fait ensuite la place à une phase de stabilisation puis de réduction des impacts, tandis que la croissance économique profitait à un nombre grandissant de personne. Las ! Bien documenté, Hervé Kempf a soutenu toutes les critiques, l'innocence des riches n'a pas été démontrée, pas plus que la tendance des économies à se dématérialiser ou la capacité de la croissance à provoquer une réduction du nombre de pauvres. Comme l'a rappelé Sylvia Perez-Vitoria, la croissance c'est surtout la croissance des inégalités et de l'exploitation de la majorité par une minorité de plus en plus riche. Le journaliste Brice Couturier s'est énervé, pendant que Mme Kosciusko-Morizet s'en remettait très largement aux progrès de la science et de la technologie, même si elle a appelé de ses vœux une croissance plus propre, « faite de modération ».

Le débat témoigne de l'embarras des élites devant la question écologique. Les vieux concepts, par lesquels la crise globale est arrivée, semblent impuissants. La décroissance met les pieds

\footnotetext{
14 http://www.decroissance.org/index.php?chemin=textes/Un_mot-obus.htm

15 Journaliste au Monde, auteur de «Comment les riches détruisent la planète » paru chez Seuil en 2006.

16 Députée UMP de l'Essonne.

17 Economiste et sociologue, membre de «La ligne d'Horizon », auteure du « Retour des paysans » chez Actes Sud.
} 
dans le plat, comme le rapport du MIT comme au Club de Rome avant elle ${ }^{18}$. Alors que la croissance est invoquée partout comme la solution à tous les problèmes, du Nord comme du Sud, un véritable sésame universel, elle affirme une conclusion scandaleuse et pourtant parfaitement fondée, à savoir d'une part que la croissance n'a pas d'avenir puisqu'elle ne résout pas le problème écologique, et d'autre part qu'elle n'a pas l'universalité qu'on lui prête.

La croissance est le résultat d'un ensemble de politiques et d'initiatives. Peu de pays, dans le monde, connaissent un ensemble de politiques et d'initiatives tels qu'il se produit une croissance accompagnée d'une réduction des inégalités. C'est plutôt le contraire. Et à l'échelle historique la chose est encore plus manifeste. Les pays qui ont connu un fort taux de croissance depuis longtemps sont ceux qui ont l'empreinte écologique la plus élevée, à quelques nuances près. La « dématérialisation du PIB » n'est qu'apparente, car les pays les plus pollueurs ont largement délocalisé les maillons les plus destructeurs de la chaîne de production qui sert à entretenir leur niveau de vie. La Chine et le Brésil ne se privent pas de rappeler qu'une grande partie des impacts écologiques de leur pays n'est que la conséquence directe de la demande gargantuesque des pays occidentaux. Et même si les problèmes écologiques n'existaient pas, la pollution mentale engendrée par le productivisme, dont le symbole suprême est la croissance, devraient poser question. La décroissance désigne alors la dés-économisation des esprits, ce qui revient à reprendre à nouveaux frais le programme du MAUSS $^{19}$.

En effet ces questions ne sont pas entièrement nouvelles. Elles sont pour la plupart issues du mouvement écologiste. Force est de constater, toutefois, que « les écolos » n'ont pas réussi à séduire. Ils ont pourtant tout essayé : écologie de droite, de gauche, ni droite ni gauche, écologie associative, écologie entrepreneuriale. Rien n'y fait, les enjeux s'aggravent et le reste de la société trouve toujours des raisons pour renvoyer les enjeux écologiques à un imaginaire écologiste qu'ils imaginent détachés de la réalité - et cela malgré les innombrables rapports tous plus officiels les uns que les autres. Catherine Larrère parle de « sociocentrisme $»^{20}$ à propos de cette étonnante incapacité de nos sociétés à voir autre chose dans la crise

18 Là aussi la traduction est instructive : le rapport original s'intitulait « The limits to growth », que Fayard avait traduit par " Halte à la croissance ». Le rapport 2003 du Club de Rome endosse toujours les conclusions du rapport de 1972, voir D. Meadows, J. Randers \& D. Meadows, Limits to growth - The 30-years update, Chelsea Green Publishing Company, 2004.

19 A. Caillé, Critique de la raison utilitaire - Manifeste du Mauss, Paris, La Découverte, 2003, Ed. orig., 2003. Le Mauss (Mouvement Anti-Utilitariste en Sciences Sociales) a été fondé en 1981.

20 C. \& R. Larrère, Du bon usage de la nature, Paris, Aubier, 1997. 
environnementale que ses problèmes relationnels. L'écologie serait avant tout le problème des écologistes. Télérama, s'étonnant du vide intellectuel existant dans le domaine de l'écologie ${ }^{21}$, témoignait autant d'une réalité tangible que de sa propre ignorance en la matière, car les théoriciens existent bel et bien. Nos sociétés semblent devoir toujours trouver une raison pour ne pas prendre au sérieux les enjeux écologiques. «Les écolos » ont été trop écologistes, puis trop « sociaux » quand ils se sont mis à prendre en compte les enjeux sociaux, bref jamais « comme il faut ». C'est que l'écologie continue de déranger, et face aux idées qui dérangent, la politique de l'autruche voire l'obscurantisme restent des recettes éprouvées.

La gamme, très large, des opposants de gauche à la décroissance donne une idée de l'importance politique et théorique des problèmes que ce terme soulève sur le plan conceptuel et politique. L'idée de décroissance est insupportable à tous ceux et toutes celles qui voient dans la médiation marchande le lien social par excellence, qu'on se fonde sur le contrat ou sur la planification. Pour beaucoup, c'est la modernité qui est en cause. C'est peut-être cela, en effet. Mais il faudrait aller plus en avant et démontrer que la modernité ainsi entendue a encore quelque chose à apporter au monde, ou plus exactement à la planète. La modernité est trop souvent portée en étendard sans plus d'argument, tout ce qui s'y oppose étant réputé réactionnaire ou obscurantiste. L'écologie pose depuis le début la question de savoir comme «ne plus être progressiste sans devenir réactionnaire » comme le dit la jolie formule en titre de l'ouvrage de Jean-Paul Besset ${ }^{22}$. La décroissance révulse la plupart des économistes - et remet en cause leur domination. Les économistes sont déjà depuis longtemps en mauvais termes avec les écologistes. A partir du moment où l'on sort des procès d'intention, l'intérêt de ces échanges est que la décroissance résiste très bien à toutes les attaques. Les objections soulevées sont familières aux écologistes : la décroissance serait relativiste (J.-M. Harribey ${ }^{23}$ ), elle serait un « retour en arrière », un retour de l'obscurantisme, une idéalisation de la nature et des sociétés « traditionnelles $»^{24}$ et bien sûr chantage à l'emploi.

21 W. Zarachowicz, Ecolos cherchent intellos, Télérama n 2970 - 16 Décembre 2006.

22 J.-P. Besset, Comment ne plus être progressiste, sans devenir réactionnaire, Paris, Fayard, 2005.

23 J.-M. Harribey, Une conception de la richesse non-marchande pour sortir du faux dilemme croissance / décroissance, E. Berr \& J.-M. Harribey (dir.), Le développement en question(s), Presses Universitaires de Bordeaux, 2006, pp. 265-288.

24 G. Duval, Décroissance ou développement durable?, Alternatives économiques, Hors-Série "Le développement durable », $1^{\text {er }}$ trimestre 2005, pp. 53-55. 
Les réponses existent des sont robustes, c'est pour cette raison que le débat perdure. La décroissance confond-t-elle «développement» et "croissance », comme le suggère René Passet ${ }^{25}$ et bien d'autres ? Et si c'était les économistes qui confondaient « bien commun » et «développement», ce dernier n'étant jamais qu'une vision réductrice, étroitement économique, du bien commun ? Bien que Passet insiste sur le qualitatif, il n'existe pas, et pour cause, de théorie économique du qualitatif. Dès lors comment situe-t-il son apport au débat ? Cela reste flou. Mettre la gestion du bien commun entre les mains calculatrices et matérialistes de l'économie, c'est s'assurer qu'on en restera au niveau de la gestion, et qu'on ne s'élèvera jamais jusqu'au niveau des projets. Il y a généralement une téléologie sousjacente aux analyses développementistes, d'ailleurs Passet reprend une nouvelle fois l'exemple de l'enfant qui grandit pour expliquer la différence entre croissance et développement. Les pays sous-développés sont-ils des enfants? Des attitudes aussi paternalistes semblaient avoir été reléguées dans les poubelles de l'histoire. Comment ne pas voir que cette analogie est un schème structurant du développement, un biologisme sousjacent qui devrait être dénoncé avec la même vigueur que certains analystes mettent à débusquer l'idée de nature dans les discours écologistes ? De celui qui dénonce sans cesse le droit naturel et celui qui recourt au développement, le plus naturaliste des deux n'est peut-être pas celui qu'on croit. Les partisans de la décroissance peuvent donc rétorquer à René Passet que c'est lui qui se trompe d'adversaire, en accréditant la thèse naturaliste dominante. En effet, reprendre le terme « développement » peut être assimilé à un soutien aux pratiques qui accompagnent ce terme. A partir d'un tel naturalisme, comment penser l'ouverture sur le monde ? Comment rouvrir notre imaginaire? Ces questions, qui sont au fondement de la critique issue de la décroissance, ne sont pas prises au sérieux, les objections tombent donc un peu à plat.

Là aussi, la critique a été faite et bien faite par le MAUSS. Le discours économique est un discours sur les moyens, qui ne laissent guère de place aux débats sur les fins. En refusant de laisser la place au discours sur les fins, les économistes bloque le libre-exercice du politique. Ils font comme s'ils avaient déjà les réponses. Faisons un sondage : la différence entre croissance et développement est-elle parfaitement claire dans l'esprit du public ? Gageons que non. En tout cas il est clair que le monde médiatique ne fait pas la différence et identifie l'un à

25 R. Passet, Querelles byzantines autour de la croissance - 
l'autre. C'est de vouloir faire la différence entre les deux qui relève de la «querelle byzantine ", même si les experts sont d'accord entre eux pour faire la leçon au bon peuple qui n'y comprend rien. Pour faire une différence entre développement économique et bien commun, le recours à un terme qui différencie clairement les deux est nécessaire.

La décroissance n'est pas une doctrine unifiée. De vives tensions entre le journal $L a$ Décroissance et l'actuel comité de rédaction d'Entropia ont accompagné l'émergence de la revue théorique. La création du PPLD n'a pas fait l'unanimité, c'est le moins qu'on puisse dire, et des tensions sont apparues dès le début dans noyau de direction. Désaccord sur l'usage du terme « décroissance » comme mot d'ordre ou comme intitulé de programme politique. La « décroissance de l'empreinte écologique » adoptée par les Verts n'a pas du tout la même portée que la décroissance tout court, c'est un choix bien différent qui a été fait. D'autres préfèrent l'après-développement, d'autres veulent éviter les slogans (Sylvia Perez-Vitoria), d'autres préfèrent la décroissance soutenable (Vincent Cheynet et Bruno Clémentin).

Au-delà de l'écume de la tempête qui agite les esprits, nous pensons qu'il est possible de caractériser la décroissance comme se situant à la confluence de cinq sources qui se croisent sans être forcément concourantes ni même convergentes.

La première est la source culturaliste. Issue de l'anthropologie, emmenée par Serge Latouche, marxiste reconverti, sa thèse principale est que l'homo economicus est contingent, dépendant de sa représentation du monde et de l'histoire. Si l'on veut ouvrir de nouveaux possibles, il est nécessaire de réinventer ou redécouvrir notre nature. Cette thèse se distingue de la thèse marxiste qui reste encore aujourd'hui très largement dépendante de l'imaginaire économiste et productiviste. La décroissance représente un moyen de prendre le contre-pied du développement sur le plan des «significations imaginaires sociales», pour utiliser le vocabulaire de Castoriadis ${ }^{26}$, et ainsi d'atteindre la société instituée pour la faire évoluer, pour ouvrir une brèche, un passage à la société instituante. Serge Latouche affirme que «l'autre monde possible» est dans celui-là, qu'il faut "décoloniser notre imaginaire » les significations économiques et développementistes pour parvenir à le voir ${ }^{27}$.

26 C. Castoriadis, L'institution imaginaire de la société, Paris, Seuil, 1975.

27 S. Latouche, Survivre au développement, Paris, Mille et Une Nuits, 2004. S. Latouche, Décoloniser l'imaginaire - la pensée créative contre l'économie de l'absurde, Lyon, Parangon, 2005. Voir aussi F. Partant, La fin du développement - la naissance d'une alternative ?, Paris, Actes Sud, 1997 ou G. Rist, Le développement - Histoire d'une croyance occidentale, Paris, Presses de Sciences Po, 1996. 
La source démocratique est issue des analyses d'Ivan Illich. Composante majeure de l'approche de Vincent Cheynet, elle s'appuie sur l'effondrement des liens sous le poids de la marchandisation. Dès lors ce qui compte est de revitaliser les liens, ce qui passe par une relocalisation qui n'est pas un retour au bon vieux temps des villages, dont on sait qu'il n'était pas toujours si bon que ça. Cette source est attachée à la qualité du débat public, et se différencie ainsi nettement des analyses marxistes qui tendent à faire du symbolique un simple reflet des rapports de production, même si la revendication d'un revenu maximal admissible montre bien que le souci de justice sociale est présent. L'abolition des privilèges est un enjeu majeur de démocratisation de nos sociétés qui ne peut utiliser des moyens qui ne soient pas en eux-mêmes démocratique sans simultanément aller à l'encontre des principes énoncés. Ici la décroissance est un terme utilisé pour provoquer des débats, c'est là une vertu performative du «mot-obus », ce qui revitalise la passion nécessaire à l'existence d'un espace public. La décroissance est aussi une conséquence économique logique de cette revitalisation qui doit se traduire par un fort ralentissement économique. En effet discuter prend du temps, aussi les relations marchandes qui s'étendent dans l'espace sans se soucier de l'existence d'un espace public fort pour les soutenir et les domestiquer devraient-elles être fortement limitées voire même interdites car relevant d'un comportement de « passager clandestin ».

La troisième source est écologiste, elle est attachée aux écosystèmes et au respect du vivant. Pour cette tendance, la décroissance est la conséquence inévitable de toute politique écologique prise au sérieux. Renforcer la productivité écologique au détriment de la productivité économique conduira à une réduction de la mécanisation qui elle-même se traduira par une diminution du PIB. L'indicateur empreinte écologique montre que le monde utilise $125 \%$ des capacités de renouvellement de la Terre ${ }^{28}$, le Rapport du Millénaire sur les Ecosystèmes montre que $60 \%$ des écosystèmes sont dégradés ou utilisés de manière non durable $^{29}$. Un récent rapport de la FAO indique que si les prélèvements continuent de s'intensifier au rythme actuel alors les océans seront épuisés, toutes pêches confondues, en $2048^{30}$. La désertification touche $1 / 3$ des terres mondiales, la diversité biologique domestique s'est effondrée de 50 à $75 \%$, les espèces disparaissent à un rythme 100 à 1000 fois plus

28 WWF, Rapport planète vivante, 2006.

29 Millenium Assessment Report, Living Beyond Our Means : Natural Assets and Human WellBeing, 2004. http://www.millenniumassessment.org / www.maweb.org

30 B. Worm \& al., Impacts of Biodiversity Loss on Ocean Ecosystem Services, Science, 3 nov 2006, vol. 314, pp. 787-790. 
rapide que le rythme préindustriel etc. Tous les signes de dégradation rapide et massive des écosystèmes sont là. Or les écosystèmes sont tout ce que nous auront quand les ressources fossiles et épuisables auront disparu. Nous avons donc largement creusé la dette écologique envers les générations à venir. L'argument écologiste ne consiste pas en un argument comptable mais en un nouveau rapport à la nature, un rapport qui ne serait plus basé sur l'exploitation mais sur le respect et la coévolution. L'homme a dominé la nature, il doit maintenant non pas s'y soumettre mais admettre qu'il n'est pas le centre de tout. L'écologie a développé des analyses proches du courant précédent sous la forme «d'écologie de l'esprit $»^{31}$, « écosophie $»^{32}$, « contrat naturel $»^{33}$ ou encore «d'écomunicipalisme $»^{34}$. La décroissance c'est aussi une décroissance de l'emprise sur la nature, qui est réputée ne pas nous appartenir et par conséquent devoir être soustraite à l'appropriation.

La quatrième source est liée à la crise de sens que traversent les sociétés industrialisées. Qui dit crise de sens dit ouverture spirituelle, vie de l'esprit, et c'est cette démarche qui a conduit Pierre Rabhi ${ }^{35}$, par exemple, vers la décroissance. Le thème qui est développé ici est le nonsens d'une vie passée à courir le «toujours plus » (de titres, d'argent, de choses) alors que l'harmonie avec la nature, les humains et le vivant en général passe plutôt par le recueillement et l'écoute. La révolution intérieure est un préalable nécessaire pour mettre fin aux dérèglements croissants qui agitent le monde. L'action non-violente et déterminée, dans la lignée des préceptes de Gandhi, est ici la seule manière de rétablir un progrès qui ne soit plus celui des forces aveugles de la technique. La simplicité volontaire, après François d'Assise, n'est pas une manière de se priver mais une façon de s'alléger afin de laisser venir à soi un sens plus profond, moins superficiel que celui qui anime l'incessant ballet des choses ordinaires. Là où la pauvreté retrouve une dignité, la misère est chassée ${ }^{36}$ et les personnes peuvent se mettre en marche pour un avenir meilleur.

La dernière source peut être dite «bioéconomiste ». C'est une source ancienne, comme les autres du reste, mais quelques auteurs sont considérés comme ayant remis la question à l'ordre du jour. Si l'écologie part du vivant, la bioéconomie parle d'organisation humaine

31 G. Bateson, Vers une écologie de l'esprit, Seuil, 1990, Ed. orig. 1972.

32 F. Guattari, Les trois écologies, Paris, Galilée, 1989.

33 M. Serres, Le contrat naturel, éd. François Bourin, Paris, 1990.

34 M. Bookchin, Pour un municipalisme libertaire, Lyon, Atelier de Création Libertaire, 2003

35 P. Rabhi, Du Sahara aux Cévennes, Paris, Albin Michel, 2002.

36 M. Rahnema, Quand la misère chasse la pauvreté, Paris, Actes Sud, 2004. 
ayant à gérer des contraintes telles que les limites des écosystèmes (« capacité de charge ») et le caractère épuisable de certaines ressources. Avec le Club de Rome, c'est Nicholas Georgescu-Roegen qui peut être considéré comme un précurseur, lui qui écrivait en 1971 que la fin de la phase industrielle sera d'autant plus rapide que le niveau de développement économique atteint est élevé ${ }^{37}$. Chaque voiture produite l'est au prix des voitures à venir ${ }^{38}$, chaque arme fabriquée est un soc de charrue en moins. La décroissance est inévitable, c'est une conséquence géologique, comme le dit Yves Cochet $^{39}$. Il s'agit désormais de gérer la rareté, l'économie devient une «gestion normative sous contrainte $»^{40}$. Georgescu-Roegen montre, comme bien d'autres économistes avant lui, que nous devons nous tourner de toute urgence vers les ressources renouvelables car elles sont les seules à pouvoir assurer l'avenir, les autres devant s'épuiser quels que soient les progrès accomplis par la science et la technologie. Mais les ressources renouvelables sont elles aussi limitées, c'est pourquoi Hermann Daly, l'un des fondateurs d'Ecological Economics, a montré il y longtemps que l'enjeu de la soutenabilité de l'économie relève aussi d'une question de taille, et pas seulement de composition ${ }^{41}$. L'économie écologique est fondamentalement ambiguë : s'agit-il d'écologiser l'économie ou d'économiser l'écologie ? Dans tous les cas, le vivant et les écosystèmes sont appréhendés sur un mode instrumental. Les limites qui sont posées peuvent être techniques ou éthico-politiques. L'écologie peut être utilisée pour exploiter plus rapidement la nature, comme pour en protéger les ressources.

Les cinq approches conduisent de manière relativement indépendante à la conclusion que la décroissance est l'un des éléments incontournables d'un avenir meilleur. Au-delà, il existe des tensions entre ces cinq approches, qui se retrouvent dans les échanges entre partisans de la décroissance comme entre partisans et opposants, mais le fait qu'elles se retrouvent autour de ce terme est remarquable et témoigne bien de la centralité du concept de « croissance », qui joue un rôle sociétal bien plus étendu que la simple définition comptable derrière laquelle les comptables nationaux se retranchent pour maintenir leur objectivité. Une bonne partie de ces débats est connue de l'écologie politique : la décroissance est-elle de droite ou de gauche ? Se réduit-elle à la simplicité volontaire (les «petits gestes ») ? etc. Les fins connaisseurs n'y verront qu'un bis repetita, la décroissance étant de toute évidence l'une des premières

37 N. Georgescu-Roegen, La décroissance, Sang de la Terre, 1994, Ed. orig. 1971, p. 66.

38 Ibid., p. 67

39 Y. Cochet, Pétrole apocalypse, Paris, Fayard, 2006.

$40 \quad$ R. Passet, L'économique et le vivant, Paris, Payot, 1979.

41 H.E. Daly \& J. Cobb Jr., For the Common Good, Boston, Beacon Press, 1989. 
conséquences quand on prend l'écologie ou la sortie de l'économisme au sérieux. Pourtant le terme a au moins partiellement réussi à régénérer un débat qui semblait enlisé.

Il est vrai que l'actualité a bien aidé. C'est en effet au début des années 2000 que la question des changements climatiques semble enfin être prise au sérieux. Le Rapport Stern ${ }^{42}$, les rapports du $\mathrm{GIEC}^{43}$, le rapport du Millénaire sur les Ecosystèmes ${ }^{44}$ et bien d'autres rapports ont une nouvelle fois démontré que les choses empirent. Même si des secteurs nonnégligeables de l'humanité connaissent une richesse jusque-là inconnue, les inégalités augmentent et les bases matérielles de cette richesse fondent comme neige au soleil.

La décroissance est devenue un élément symbolique incontournable. Les Echos, journal peu susceptible de fantaisie sur le plan économique, a fait de la décroissance l'un des trois paradigmes économiques susceptibles de nous sortir du «prêt-à-penser » dans ce domaine ${ }^{45}$. La gauche, en mal d'idées neuves, devrait se pencher sur le sujet. On a vu des comités « Désirs d'avenir » engager des discussions publiques autour de la décroissance. Ira-t-elle jusqu'au bout?

42 Stern, Stern Review on the Economics of Climate Change, 2006.

43 IPCC, Summary for policymakers - working group 1, 2007.

44 Op.cit.

45 Sortir du prêt-à-penser économique, décembre 2004. 